\title{
I Macroeteroceri della Riserva Naturale Orientata "Laghetti di Marinello" (Sicilia nord-orientale) (Lepidoptera Heterocera)
}

\begin{abstract}
Riassunto - Gli scopi di questa ricerca sono quelli di contribuire alla conoscenza della macrolepidotterofauna della Sicilia nord-orientale e di caratterizzare la comunità presente nella Riserva Naturale Orientata "Laghetti di Marinello". Sono stati effettuati 23 campionamenti tra dicembre 2007 e dicembre 2008 col metodo della caccia al lume. Sono stati catturati 2.537 individui appartenenti a 160 specie, tra cui Chloroclystis v-ata e Laelia coenosa, segnalate per la prima volta in Sicilia; importanti anche le segnalazioni di Calamodes subscudularia, Mythimna languida e M. joannisi, specie piuttosto rare e localizzate. Le specie più abbondanti sono state: Eilema caniola, Eublemma viridula, Idaea filicata, Dysauxes famula e Idaea seriata, che da sole rappresentano il $42 \%$ di tutta la comunità, favorite dalla presenza delle rispettive piante nutrici e dall'elevato adattamento alle condizioni climatiche del sito. Il numero di specie e i valori degli indici di diversità indicano un basso livello di biodiversità ospitata, ma la fenologia, le analisi corologica e autoecologica delle specie, insieme ai confronti effettuati con altre faune, indicano una comunità ben caratterizzata e peculiare, diretta espressione delle condizioni abiotiche e biotiche della Riserva.

Abstract - Macroheterocera of the Oriented Natural Reserve "Laghetti di Marinello" (north-eastern Sicily) (Lepidoptera: Heterocera). The aim of this research is to contribute to the knowledge of the fauna of macrolepidoptera of north-eastern Sicily and to describe the community present in the Oriented Natural Reserve "Laghetti di Marinello". 23 samples were carried out from December 2007 to December 2008 using a white vertical sheet reflecting a light source. 2.537 specimens belonging to 160 species were captured. Chloroclystis v-ata and Laelia coenosa were reported for the first time in Sicily, Calamodes subscudularia, Mythimna languida and M. joannisi, very rare and localized species, were also reported. The most abundant species were: Eilema caniola, Eublemma viridula, Idaea filicata, Dysauxes famula and Idaea seriata, which represent the $42 \%$ of the whole community and are favoured by the presence of their feeding plants and by their high adaptability to the climatic conditions of the place. The number of species and the values of the diversity indices show a low level of biodiversity, but phenology, chorological and autoecological analysis of the species, with comparisons made with other faunae, show a well characterized and peculiar community, direct expression of the abiotic and biotic conditions of the Reserve.
\end{abstract}

Key words: Aree umide, aree rifugio, fauna, biodiversità, Sicilia

\section{INTRODUZIONE}

Fra le regioni meridionali, la Sicilia è certamente quella maggiormente studiata dai lepidotterologi, soprattutto a causa delle sue particolarità ambientali e biogeografiche, come attestano le numerose pubblicazioni aventi come oggetto i Macrolepidotteri dell'isola (Schwingenschuss, 1942; Mariani, 1939, 1948; Parenzan \& Hausmann, 1992; Hausmann, 1993; Giuliano \& Parenzan, 1994; Bella et al., 1995; Grillo \& Parenzan, 1995, 1996; Cernigliaro et al., 1996; Russo et al., 2001; Parenzan et al., 2002; Parenzan et al., 2006, solo per citarne alcuni). Fa eccezione l'area nord-orientale, specialmente la provincia di Messina, per la quale esistono solo sporadiche segnalazioni, spesso datate (Heeger, 1838; Lederer, 1852; Mariani, 1939; Zeller, 1847). In ogni caso si tratta, per la maggior parte, di ricerche meramente faunistiche, comprendenti diverse località e ambienti eterogenei.

Molto poche sono anche le ricerche riguardanti le zone umide costiere. Vista la scarsa biomassa ve- getale presente e, di conseguenza, la scarsa biodiversità zoologica ospitata, raramente l'attenzione degli entomologi si è concentrata su queste aree, nonostante esse rappresentino importanti "ambienti rifugio" per numerose specie. In questo quadro, sono particolarmente significativi i lavori di Parenzan (1998) e di Bella et al. (1999) che, riguardando miratamente proprio questo tipo di habitat (rispettivamente la Riserva Naturale Orientata di Vendicari e il Pantano di Longarini), forniscono importanti dati sulle comunità, utilizzabili per confronti fra ambienti simili anche sul lungo periodo. A questi si aggiungano le recenti segnalazioni faunistiche riguardanti proprio la Riserva Naturale Orientata "Laghetti di Marinello" (Infusino, 2008; Infusino et al., 2010), oggetto del presente studio.

Lo scopo di questa ricerca è quello di dare un contributo alla conoscenza dei Macroeteroceri della Sicilia nord-orientale e, nel dettaglio, di caratterizzare la comunità presente nella Riserva Naturale Orientata "Laghetti di Marinello", una delle ultime aree umide costiere ancora presenti sull'isola. 


\section{Area di STUdiO}

La Riserva Naturale Orientata "Laghetti di Marinello" si trova lungo la costa tirrenica, nella parte settentrionale dei monti Peloritani, ai piedi di Capo Tindari ( $38^{\circ} 8^{\prime} 26^{\prime \prime} \mathrm{N} ; 5^{\circ} 3^{\prime} 2^{\prime \prime}$ E). Si estende su una superficie di circa 378 ettari e rappresenta uno degli ultimi esempi di ambiente salmastro costiero presenti nella Sicilia nord-orientale (fig. 1).

Geologia. La riserva è costituita da rocce metamorfiche che si sono formate all'incirca tra 370 e 200 milioni di anni fa, nel Mesozoico e Paleozoico. Dal punto di vista geo-morfologico, il sistema lagunare di Marinello ha un'origine alluvionale recente, dovuta allo sviluppo, per oltre $1 \mathrm{~km}$ in direzione SE, di distese sabbiose litorali (Olocene Attuale) che hanno determinato la chiusura di un corrispondente tratto di mare. Anche se l'origine dei laghetti risale a circa 100 anni fa, come testimonia la cartografia IGM del 1895, sono state le opere di disboscamento effettuate negli anni '30 lungo il Torrente Timeto a provocare un aumento di trasporto di detriti verso la fascia costiera, dove le condizioni locali (correnti marine, conoide sottomarina, venti) hanno favorito la sedimentazione e la formazione di tali distese. L'area viene continuamente rimodellata, con variazioni morfologiche della linea della spiaggia e delle dimensioni dei laghetti; il fenomeno si manifesta quando le onde del mare dominante, incidendo in maniera radente rispetto alla costa, in corrispondenza di un improvviso e netto approfondimento dei fondali che provoca la rifrazione delle onde, determinano il deposito dei sedimenti del trasporto litoraneo sotto forma di cordoni dunali. Inoltre, la costruzione delle gallerie autostradali ha provocato il deposito di ulteriore materiale solido con conseguente ampliamento della lingua di sabbia. Attualmente esistono cinque laghetti semi-permanenti, tre più interni, Lago Marinello, Lago Mergolo della Tonnara e Lago Verde, due esterni, Porto Vecchio e Fondo Porto. Le lagune interne hanno acque dolci e salmastre, mentre quelle più vicine al mare sono caratterizzate da una maggiore salinità. Alle spalle del sistema dunale è presente una scogliera alta 250 m s.l.m., con pareti verticali a picco sul mare, costituita da marmi Ercinici a biotite e muscovite appartenenti al basamento cristallino dell'Unità stratigrafico-strutturale Alpina del Mela (Monti Peloritani Settore Meridionale dell'Arco Calabro-Peloritano). Per i peculiari caratteri geo-morfologici, la Riserva "Laghi di Marinello" è considerata un Geosito (Infusino et al., 2008).

VegetAZIONE. La zona è caratterizzata da una notevole ricchezza floristica ed è possibile identificare otto associazioni vegetali.

Macchia a euforbia arborescente (Oleo - Euphorbietum dendroidis): la specie caratteristica e dominante di questa associazione è Euphorbia den-

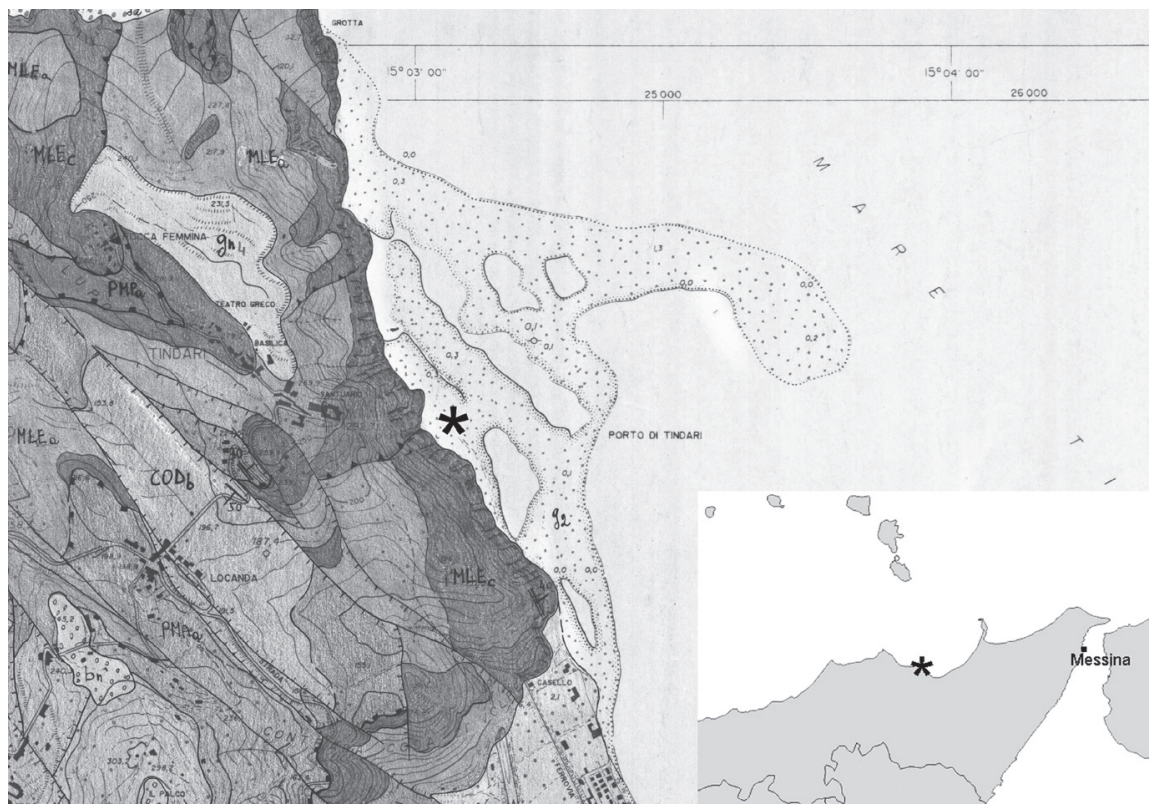

Fig. 1. Individuazione dell'area di studio e posizionamento della lampada $(*)$. 
droides L., alla quale si associano le sclerofille Pistacia lentiscus L. e Olea europea L. var. sylvestris (Mill.) Lehr, costituendo la tipica macchia mediterranea termo-xerofila.

Praterie steppiche perenni a taglia mani e caglio dell'Etna (Galio aetnici - Ampelodesmetum mauritanici): quest'associazione è caratterizzata da Galium aetnicum Biv. e Ampelodesmos mauritanicus (Poir.) Dur. \& Schinz, che lasciano poco spazio ad altre specie perenni.

Praterie steppiche perenni a barboncino mediterraneo (Ferulo - Hyparrhenietum hirtae): caratterizzate da Ferula communis L. e dominate da Hyparrhenia hirta (L.) Stapf e Andropogon distachyos L.

Vegetazione arbustiva a tamerici (Tamerici africanae - Viticetum agni-casti): associazione caratterizzata dalla presenza di Tamarix africana Poir e Vites agnus-castus L. A queste specie si associano Nerium oleander L. e Rubus ulmifolius Schott.

Cespuglieti pionieri dei substrati incoerenti (Scrophulario - Helichrysetea): la classe Scrophulario-Helichrysetea comprende piccoli arbusti che colonizzano substrati incoerenti (ghiaie) ed è caratterizzata da Helichrysum italicum subsp. siculum (Jord. \& Fourr.) Galbany et al. e Scrophularia canina L. subsp. bicolor (Sm.) Greuter. Sull'arenile della Laguna di Oliveri-Tindari è presente un'associazione tipica che è stata chiamata Echinopo spinosissimi-Helichrysetum italici, dominata da Helichrysum italicum subsp. siculum (Jord. \& Fourr.) Galbany et al. e Echinops spinosissimus Turra subsp. spinosissimus.

Vegetazione rupicola casmofitica perenne naturale (Asplenietalia glandulosi): nell'ordine Asplenietalia glandulosi rientrano diverse fitocenosi che crescono sulle pareti rocciose della Riserva. Le specie caratteristiche sono: Ballota rupestris (Biv.) Vis., Ficus carica L., Lomelosia cretica (L.) Greuter \& Burdet e Teucrium flavum L. A queste si associano importanti paleo endemismi rupicoli come Dianthus rupicola subsp. aeolicus (Lojac.) Brullo \& P. Minissale, Centaurea panormitana subsp. seguenzae (Lacaiata) Greuter, Hyoseris lucida subsp. taurina (Pamp.) Peruzzi \& Vangelisti, Brassica incana Ten. e Matthiola incana subsp. rupestris (Raf.) Nyman. È stata proposta da Guarino la sub-associazione centau- retosum seguenzae che risulta esclusiva del promontorio di Tindari, in quanto ha come specie caratteristica la già citata Centaurea panormitana subsp. seguenzae (Lacaiata) Greuter, un endemismo esclusivo di quest'area.

Vegetazione a elofite (Phragmitetum communis): quest'associazione paucispecifica è caratterizzata da Phragmites australis (Cav.) Trin. che si insedia su suoli periodicamente inondati.

Vegetazione alo-igrofila (Juncetum maritimi-acuti): caratterizzata dalla presenza di Juncus acutus L. (Licandro et al., 2011).

Clima. Pur essendo la provincia di Messina la più piovosa dell'isola, la nostra area di studio ricade nella zona costiera, caratterizzata da un'accentuata aridità, specialmente nel periodo primaverile-estivo: le temperature medie annue sono di $18-19^{\circ} \mathrm{C}$, con medie mensili che variano fra 11 e $25^{\circ} \mathrm{C}$, mentre le precipitazioni medie sono paragonabili al resto della Sicilia e cioè circa $740 \mathrm{~mm}$ annui, con un minimo in agosto di soli $9 \mathrm{~mm}$ e un massimo a dicembre di 111 mm (Cartabellotta et al., 1998).

\section{MAteriali e Metodi}

Oggetto del presente studio sono le specie appartenenti alle superfamiglie Cossioidea, Bombycoidea, Drepanoidea, Geometroidea, Noctuoidea, nel loro complesso considerate Macroeteroceri.

Il campionamento è stato effettuato tramite l'utilizzo di una lampada a luce miscelata da $160 \mathrm{~W}$ posta a $20-30 \mathrm{~cm}$ da un telo bianco di circa $1,80 \mathrm{~m}$ di altezza e $2 \mathrm{~m}$ di larghezza, a un'altezza di 1,50 m, alimentata da un generatore portatile. Il prelievo degli esemplari è avvenuto direttamente sul telo e/o a terra; per quelli individuati in volo è stato utilizzato un retino.

Sono stati effettuati 23 campionamenti fra dicembre 2007 e dicembre 2008 con una cadenza bisettimanale (per le condizioni meteorologiche particolarmente avverse, non è stato possibile effettuare un secondo campionamento nel mese di febbraio). In accordo con altre ricerche simili a quella in oggetto (Scalercio \& Infusino, 2003, 2006; Scalercio et al., 2008b), i campionamenti hanno interessato le prime 4 ore della scotofase; tali modalità di campionamento, infatti, risultano essere particolarmente efficaci per caratterizzare una comunità (Scalercio et al., 2008a). 
Gli indici di similarità di Sørensen e di Jaccard sono stati calcolati utilizzando un apposito foglio di lavoro Excel. Una stima del numero di specie potenzialmente ospitato dalla comunità è stata ottenuta utilizzando gli indici $\hat{\mathrm{S}}_{\text {Chaol }}$ e $\hat{\mathrm{S}}_{\text {Chao2 }}$ (Lee \& Chao, 1994), calcolati tramite il software Estimates 7.5.0, che è stato utilizzato anche per il calcolo degli indici di diversità.

Sul materiale raccolto è stata condotta un'analisi corologica sia qualitativa che quantitativa, utilizzando i corotipi di Parenzan (1994).

Alcuni esemplari di Geometridae sono stati oggetto di barcoding analysis secondo il protocollo metodologico previsto dal progetto "Global Barcoding AllGeos Campaign", parte integrante dell'“International Barcoding of Life Project - iBOL" (Ratnasingham \& Hebert, 2007), in cooperazione con il Zoologische Staatssammlung München di Monaco di Baviera (Germania) e la University of Guelph (Ontario, Canada).

\section{Risultati}

Sono state campionate 160 specie per un totale di 2.537 individui. Per ogni specie vengono riportati il corotipo sensu Parenzan (1994), i dati di cattura (dettagliati solo quando non è stato possibile descrivere una fenologia), la temperatura e l'umidità relativa minima e massima alla quale è stata raccolta. I mesi sono scritti in numeri romani. Le specie endemiche sono indicate con (E). Ulteriori informazioni sono state inserite, sotto forma di note alla fine della tabella, per le specie di particolare interesse faunistico e/o ecologico e per quelle rappresentate da più di 40 esemplari.

\begin{tabular}{|c|c|c|c|c|c|}
\hline Specie & Corotipo & $\begin{array}{c}\mathrm{N}^{\circ} \\
\text { esemplari }\end{array}$ & $\begin{array}{l}\text { Dati di } \\
\text { cattura }\end{array}$ & Temperatura & $\begin{array}{l}\text { Umidità } \\
\text { relativa }\end{array}$ \\
\hline \multicolumn{6}{|l|}{$\overline{\text { COSSIDAE }}$} \\
\hline Parahypopta caestrum (Hübner, 1808) & EUS2 & 6 & V-VI & $17-18^{\circ} \mathrm{C}$ & $80-83 \%$ Ur. \\
\hline Dyspessa aculeata Turati, 1909 & SIC (E) & 3 & 15.V.2008 & $17^{\circ} \mathrm{C}$ & $83 \%$ Ur. \\
\hline \multicolumn{6}{|l|}{ SPHINGIDAE } \\
\hline Marumba quercus (D. \& S., 1775) & MES4 & 1 & 07.VII.2008 & $28^{\circ} \mathrm{C}$ & $47 \%$ Ur. \\
\hline Laothoe populi (Linnaeus, 1758) & CEM & 1 & 18.IV.2008 & $15^{\circ} \mathrm{C}$ & $77 \%$ Ur. \\
\hline \multicolumn{6}{|l|}{ DREPANIDAE } \\
\hline Watsonalla cultraria (Fabricius, 1775) & EUR6 & 1 & 07.VII.2008 & $28^{\circ} \mathrm{C}$ & $47 \%$ Ur. \\
\hline \multicolumn{6}{|l|}{ GEOMETRIDAE } \\
\hline Ligdia adustata (D. \& S., 1775) & CAE & 1 & 04.III.2008 & $13^{\circ} \mathrm{C}$ & $84 \%$ Ur. \\
\hline Stegania trimaculata (Villers, 1789) & CEM & 2 & 07.VII.2008 & $28^{\circ} \mathrm{C}$ & $47 \%$ Ur. \\
\hline Enconista spodiaria (Lefèbvre, 1832) & MEW & 21 & I, IV-V & $12-17^{\circ} \mathrm{C}$ & $74-98 \%$ Ur. \\
\hline Rhoptria asperaria (Hübner, 1817) & EUS1 & 2 & 18.VI.2008; 15-X-2008 & $20-22^{\circ} \mathrm{C}$ & $81-86 \%$ Ur. \\
\hline Pachycnemia hippocastanaria (Hübner, 1799) & MEE & 1 & 11.XI.2008 & $15^{\circ} \mathrm{C}$ & $76 \%$ Ur. \\
\hline Ennomos quercaria (Hübner, 1813) & EUS3 & 1 & 21.VIII.2008 & $23^{\circ} \mathrm{C}$ & $76 \%$ Ur. \\
\hline Selenia lunularia (Hübner, 1788) & CAE1 & 1 & 18.VI.2008 & $22^{\circ} \mathrm{C}$ & $81 \%$ Ur. \\
\hline Crocallis Boisduval,iaria (H. Lucas, 1849) & NAW8 & 2 & 27.IX.2008; 15.X.2008 & $19-20^{\circ} \mathrm{C}$ & $69-86 \%$ Ur. \\
\hline Nychiodes ragusaria Millière, 1884 & APS9 (E) & 1 & $30 . X .2008$ & $19^{\circ} \mathrm{C}$ & $81 \%$ Ur. \\
\hline Menophra abruptaria (Thunberg, 1792) & MED5 & 24 & I-VI, VIII-X & $9-24^{\circ} \mathrm{C}$ & $74-98 \%$ Ur. \\
\hline Menophra japygiaria (O. Costa, 1849) & MEW5 & 9 & I, IV-VII, VIII, X & $9-28^{\circ} \mathrm{C}$ & $47-86 \% \mathrm{Ur}$. \\
\hline Calamodes subscudularia (Turati, 1919) & NAF9 & 17 & $\mathrm{X}$ & $19-20^{\circ} \mathrm{C}$ & $81-86 \% \mathrm{Ur}$ \\
\hline Peribatodes rhomboidaria (D. \& S., 1775) & CEM & 11 & IV-VI, X, XII & $13-20^{\circ} \mathrm{C}$ & $67-86 \%$ Ur. \\
\hline Ascotis selenaria (D. \& S., 1775) & CAE & 1 & 18.IV.2008 & $15^{\circ} \mathrm{C}$ & $77 \%$ Ur. \\
\hline Campaea honoraria (D. \& S., 1775) & EUS1 & 1 & 04.VI.2008 & $18^{\circ} \mathrm{C}$ & $80 \%$ Ur. \\
\hline Charissa (Euchrognophos) variegata (Duponchel, 1830) & MES5 & 40 & II-VIII, X, XII & $13-28^{\circ} \mathrm{C}$ & $47-84 \%$ Ur. \\
\hline Semiaspilates ochrearia (Rossi, 1794) & MES4 & 1 & 04.III.2008 & $13^{\circ} \mathrm{C}$ & $84 \%$ Ur. \\
\hline Dyscia sicanaria (Oberthür, 1923) & TUM & 2 & 04.VI.2008; 30.X.2008 & $18-19^{\circ} \mathrm{C}$ & $80-81 \%$ Ur. \\
\hline Pseudoterpna coronillaria (Hübner, 1817) & MES & 6 & III, X-XI & $12-20^{\circ} \mathrm{C}$ & $80-86 \% \mathrm{Ur}$ \\
\hline Chlorissa etruscaria (Zeller, 1849) & TUM & 4 & IV, VI-VII & $15-28^{\circ} \mathrm{C}$ & $47-81 \% \mathrm{Ur}$ \\
\hline Microloxia herbaria (Hübner, 1813) & CAM & 44 & IV-VII & $15-28^{\circ} \mathrm{C}$ & $47-83 \% \mathrm{Ur}$ \\
\hline Cyclophora puppillaria (Hübner, 1799) & TUM+mac & 6 & I, V-VII & $12-28^{\circ} \mathrm{C}$ & $47-98 \% \mathrm{Ur}$. \\
\hline Cyclophora porata (Linnaeus, 1767) & EUR2 & 2 & 29.IV.2008 & $15^{\circ} \mathrm{C}$ & $79 \% \mathrm{Ur}$ \\
\hline Cyclophora suppunctaria (Zeller, 1847) & EUS1-4 & 2 & 27.IX.2008; 15.X.2008 & $19-20^{\circ} \mathrm{C}$ & $69-86 \%$ Ur. \\
\hline Scopula nigropunctata (Hufnagel, 1767) & ASE & 1 & 15.X.2008 & $20^{\circ} \mathrm{C}$ & $86 \%$ Ur. \\
\hline Scopula ornata (Scopoli, 1763) & PAL & 2 & 07.VII.2008; 15.X.2008 & $20-28^{\circ} \mathrm{C}$ & $47-86 \%$ Ur. \\
\hline Scopula submutata (Treitschke, 1828) & MED & 1 & 03.IV.2008 & $13^{\circ} \mathrm{C}$ & 74\%Ur. \\
\hline
\end{tabular}




\begin{tabular}{|c|c|c|c|c|c|}
\hline Specie & Corotipo & $\begin{array}{c}\mathrm{N}^{\circ} \\
\text { esemplari }\end{array}$ & $\begin{array}{l}\text { Dati di } \\
\text { cattura }\end{array}$ & Temperatura & $\begin{array}{l}\text { Umidità } \\
\text { relativa }\end{array}$ \\
\hline Scopula decorata (D. \& S., 1775) & PAL & 1 & 03.IV.2008 & $13^{\circ} \mathrm{C}$ & $74 \% \mathrm{Ur}$ \\
\hline Scopula vigilata Prout, 1913 & MEE & 1 & 06.VIII.2008 & $23^{\circ} \mathrm{C}$ & $75 \%$ Ur. \\
\hline Scopula marginepunctata (Goeze, 1781$)$ & CEM & 25 & III-X & $13-24^{\circ} \mathrm{C}$ & $69-85 \%$ Ur. \\
\hline Scopula imitaria (Hübner, 1799) & MES & 28 & III-VIII, XI & $12-28^{\circ} \mathrm{C}$ & $47-85 \%$ Ur. \\
\hline Scopula minorata (Boisduval, 1833) & $\mathrm{AFM}+\mathrm{mac}$ & 68 & VI-VII & $18-28^{\circ} \mathrm{C}$ & $47-80 \%$ Ur. \\
\hline Glossotrophia confinaria (Herrich-Schäffer, 1847) & TIR1 & 2 & 30.X.2008 & $19^{\circ} \mathrm{C}$ & $81 \% \mathrm{Ur}$ \\
\hline Idaea leipnitzi (Hausmann, 2004) & ADR9 & 10 & VI-VII & $18-28^{\circ} \mathrm{C}$ & $47-81 \%$ Ur. \\
\hline Idaea consanguinaria (Lederer, 1853) & MEE & 40 & VI & $22^{\circ} \mathrm{C}$ & $81 \%$ Ur. \\
\hline Idaea rusticata (D. \& S., 1775) & CAE1 & 13 & VI-VIII & $22-28^{\circ} \mathrm{C}$ & $47-81 \% \mathrm{Ur}$ \\
\hline Idaea filicata (Hübner, 1799) & EUS2 & 218 & IV-VI, VIII-X & $15-24^{\circ} \mathrm{C}$ & $69-86 \%$ Ur. \\
\hline Idaea attenuaria (Rambur, 1833) & MEW & 18 & IV, VI-VII, IX-X & $15-28^{\circ} \mathrm{C}$ & $47-86 \%$ Ur. \\
\hline Idaea elongaria (Rambur, 1833) & TUM & 1 & 18.IV.2008 & $15^{\circ} \mathrm{C}$ & $77 \%$ Ur. \\
\hline Idaea obsoletaria (Rambur, 1833) & TUM & 5 & VIII-IX & $23-24^{\circ} \mathrm{C}$ & $76-82 \% \mathrm{Ur}$. \\
\hline Idaea longaria (Herrich-Schäffer, 1852) & MED & 1 & 07.VII.2008 & $28^{\circ} \mathrm{C}$ & $47 \% \mathrm{Ur}$ \\
\hline Idaea seriata (Schrank, 1802) & EUM & 182 & I-XII & $9-28^{\circ} \mathrm{C}$ & $47-98 \%$ Ur. \\
\hline Idaea dimidiata (Hufnagel, 1767) & BAA & 6 & III-VI & $13-22^{\circ} \mathrm{C}$ & $77-84 \% \mathrm{Ur}$ \\
\hline Idaea camparia (Herrich-Schäffer, 1852) & TUM & 12 & IV, VII & $15-28^{\circ} \mathrm{C}$ & $47-79 \%$ Ur. \\
\hline Idaea subsericeata (Haworth, 1809) & CEM & 18 & IV, VII, IX-X & $15-28^{\circ} \mathrm{C}$ & $47-86 \%$ Ur. \\
\hline Idaea infirmaria (Rambur, 1833) & MED & 3 & VI-VII & $18-28^{\circ} \mathrm{C}$ & $47-80 \% \mathrm{Ur}$. \\
\hline Idaea distinctaria (Boisduval, 1840) & MEE & 19 & IV-V & $15-17^{\circ} \mathrm{C}$ & $77-83 \% \mathrm{Ur}$ \\
\hline Idaea degeneraria (Hübner, 1799) & CEM & 28 & IV-V, IX-X & $15-20^{\circ} \mathrm{C}$ & $69-86 \% \mathrm{Ur}$ \\
\hline Idaea deversaria (Herrich-Schäffer, 1847) & CAE1 & 1 & 04.VI.2008 & $18^{\circ} \mathrm{C}$ & $80 \%$ Ur. \\
\hline Rhodometra sacraria (Linnaeus, 1767) & $\mathrm{COS}$ & 54 & IV, VII-XI & $12-24^{\circ} \mathrm{C}$ & $69-86 \% \mathrm{Ur}$. \\
\hline Orthonama obstipata (Fabricius, 1794) & SCO & 2 & 20.XII.2007; 19.III.2008 & $10-14^{\circ} \mathrm{C}$ & $84-85 \%$ Ur. \\
\hline Xanthorhoe disjunctaria (La Harpe, 1860) & MEW & 37 & I-IV, VI, X, XII & $9-20^{\circ} \mathrm{C}$ & $67-98 \%$ Ur. \\
\hline Catarhoe basochesiata (Duponchel, 1831) & MEW & 6 & I, III, X & $12-19^{\circ} \mathrm{C}$ & $81-98 \%$ Ur. \\
\hline Epirrhoe alternata (Müller, 1764) & OLA & 2 & 03.IV.2008; 03.IX.2008 & $13-24^{\circ} \mathrm{C}$ & $74-82 \%$ Ur. \\
\hline Epirrhoe galiata (D. \& S., 1775) & TUE1 & 41 & I, III-V, X-XII & $9-20^{\circ} \mathrm{C}$ & $74-98 \%$ Ur. \\
\hline Larentia malvata (Rambur, 1833) & MEW & 1 & 15.X.2008 & $20^{\circ} \mathrm{C}$ & $86 \% \mathrm{Ur}$ \\
\hline Nebula salicata (D. \& S., 1775) & EUS2 & 28 & I-IV, X-XI & $9-20^{\circ} \mathrm{C}$ & $74-98 \%$ Ur. \\
\hline Eupithecia venosata (Fabricius, 1787) & CAE1 & 2 & 19.III.2008 - 18.IV.2008 & $14-15^{\circ} \mathrm{C}$ & $77-85 \%$ Ur. \\
\hline Eupithecia centaureata (D. \& S., 1775) & PAL & 1 & 15.X.2008 & $20^{\circ} \mathrm{C}$ & $86 \%$ Ur. \\
\hline Eupithecia semigraphata Bruand, 1850 & EUS3 & 2 & 27.IX.2008; 15.X.2008 & $19-20^{\circ} \mathrm{C}$ & $69-86 \% \mathrm{Ur}$. \\
\hline Eupithecia dodoneata Guenée, 1858 & EUR1 & 3 & 19.III.2008 & $14^{\circ} \mathrm{C}$ & $85 \%$ Ur. \\
\hline Eupithecia abbreviata Stephens, 1831 & EUR1 & 3 & IV & $15^{\circ} \mathrm{C}$ & $77 \%$ Ur. \\
\hline Gymnoscelis rufifasciata (Haworth, 1809) & PAL & 47 & I, III-X & $12-24^{\circ} \mathrm{C}$ & $47-85 \%$ Ur. \\
\hline Chloroclystis v-ata (Haworth, 1809) & ASE & 4 & III-V & $14-17^{\circ} \mathrm{C}$ & $79-85 \%$ Ur. \\
\hline \multicolumn{6}{|l|}{ NOTODONTIDAE } \\
\hline Phalera bucephala (Linnaeus, 1758) & ASE & 1 & 03.IX.2008 & $24^{\circ} \mathrm{C}$ & $82 \%$ Ur. \\
\hline Harpyia milhauseri (Fabricius, 1775) & ASE & 1 & 29.IV.2008 & $15^{\circ} \mathrm{C}$ & $79 \%$ Ur. \\
\hline \multicolumn{6}{|l|}{ NoctUIDAE } \\
\hline Nodaria nodosalis (Herrich-Schäffer, 1851) & AFM & 7 & IV-V, IX-X & $15-19^{\circ} \mathrm{C}$ & $69-83 \%$ Ur. \\
\hline Pechipogo plumigeralis (Hübner, 1825) & TEM & 3 & IV, VII-VIII & $15-23^{\circ} \mathrm{C}$ & $50-79 \%$ Ur. \\
\hline Catocala nymphagoga (Esper, 1787) & MES & 1 & 18.VI.2008 & $22^{\circ} \mathrm{C}$ & $81 \%$ Ur. \\
\hline Clytie illunaris (Hübner, 1813) & MED5 & 9 & V, VII, IX & $17-28^{\circ} \mathrm{C}$ & $47-83 \%$ Ur. \\
\hline Dysgonia algira (Linnaeus, 1767) & MES & 12 & IV-VI, VIII-X & $15-23^{\circ} \mathrm{C}$ & $69-86 \% \mathrm{Ur}$ \\
\hline Apopestes spectrum (Esper, 1787) & PAL + India & 1 & 29.IV.2008 & $15^{\circ} \mathrm{C}$ & $79 \%$ Ur. \\
\hline Aedia leucomelas (Linnaeus, 1758) & CEM & 1 & 29.IV.2008 & $15^{\circ} \mathrm{C}$ & $79 \%$ Ur. \\
\hline Zethes insularis Rambur, 1833 & MED & 7 & IV, VI, IX & $15-24^{\circ} \mathrm{C}$ & $77-82 \% \mathrm{Ur}$. \\
\hline Hypena obsitalis (Hübner, 1813) & $\mathrm{EUM}+\mathrm{mac}$ & 2 & 18.IV.2008; 15.V.2008 & $15-17^{\circ} \mathrm{C}$ & $77-83 \%$ Ur. \\
\hline Hypena lividalis (Hübner, 1796) & TUM+mac+India & 5 & III, VII-VIII, XI & $12-28^{\circ} \mathrm{C}$ & $47-85 \%$ Ur. \\
\hline Eutelia adulatrix (Hübner, 1813) & $\mathrm{TUM}+\mathrm{mac}$ & 6 & V-VIII & $17-28^{\circ} \mathrm{C}$ & 47-83\%Ur. \\
\hline Autographa gamma (Linnaeus, 1758) & PAL & 16 & I-III, VII, X-XII & $9-28^{\circ} \mathrm{C}$ & $47-98 \%$ Ur. \\
\hline Thysanoplusia orichalcea (Fabricius, 1775) & CEM & 1 & 06.II.2008 & $13^{\circ} \mathrm{C}$ & $81 \%$ Ur. \\
\hline Trichoplusia ni (Hübner, 1803) & SCO & 1 & 30.X.2008 & $19^{\circ} \mathrm{C}$ & $81 \%$ Ur. \\
\hline Ctenoplusia accentifera (Lefèbvre, 1827) & AFM & 2 & 21.XI.2007; 18.IV.2008 & $12-15^{\circ} \mathrm{C}$ & $77-80 \% \mathrm{Ur}$. \\
\hline Chrysodeixis chalcites (Esper, 1789) & SCO & 2 & 03.IX.2008; 11.XI.2008 & $15-24^{\circ} \mathrm{C}$ & $76-82 \% \mathrm{Ur}$ \\
\hline Abrostola triplasia (Linnaeus, 1758) & $\mathrm{SIE}+\mathrm{mac}$ & 1 & 29.IV.2008 & $15^{\circ} \mathrm{C}$ & $79 \%$ Ur. \\
\hline Abrostola agnorista (Dufay, 1956) & EUS & 3 & 29.IV.2008 & $15^{\circ} \mathrm{C}$ & $79 \%$ Ur. \\
\hline Pseudozarba bipartita (Herrich-Schäffer, 1850) & MED & 1 & 23.VII.2008 & $22^{\circ} \mathrm{C}$ & $50 \%$ Ur. \\
\hline Coccidiphaga scitula (Rambur, 1833) & AIM & 2 & 18.VI.2008; 21.VIII.2008 & $22-23^{\circ} \mathrm{C}$ & $76-81 \% \mathrm{Ur}$. \\
\hline
\end{tabular}




\begin{tabular}{|c|c|c|c|c|c|}
\hline Specie & Corotipo & $\begin{array}{c}\mathrm{N}^{\circ} \\
\text { esemplari }\end{array}$ & $\begin{array}{l}\text { Dati di } \\
\text { cattura }\end{array}$ & Temperatura & $\begin{array}{l}\text { Umidità } \\
\text { relativa }\end{array}$ \\
\hline Odice suava (Hübner, 1813) & EUS & 3 & VII & $28^{\circ} \mathrm{C}$ & 47\%Ur. \\
\hline Eublemma viridula (Guenée, 1841) & ESW & 227 & IV-V-X & $15-28^{\circ} \mathrm{C}$ & $47-83 \%$ Ur. \\
\hline Eublemma candidana (Fabricius, 1794) & TUM & 47 & IV-X & $15-28^{\circ} \mathrm{C}$ & $47-86 \%$ Ur. \\
\hline Eublemma ostrina (Hübner, 1808) & $\mathrm{CEM}+\mathrm{mac}$ & 15 & III-IV, VI-VII, IX-XII & $12-28^{\circ} \mathrm{C}$ & $47-85 \%$ Ur. \\
\hline Eublemma parva (Hübner, 1808) & $\mathrm{TEM}+\mathrm{mac}$ & 33 & VI, VIII-X, XII & $13-24^{\circ} \mathrm{C}$ & $67-86 \%$ Ur. \\
\hline Metachrostis velox (Hübner, 1813) & MED5 & 5 & VI, IX & $22-24^{\circ} \mathrm{C}$ & $81-82 \% \mathrm{Ur}$. \\
\hline Shargacucullia scrophulariae (D. \& S., 1775) & CEM & 13 & III-IV, & $13-15^{\circ} \mathrm{C}$ & $74-85 \%$ Ur. \\
\hline Pyrois effusa (Boisduval, 1828) & MES & 11 & I, III & $12-13^{\circ} \mathrm{C}$ & $84-98 \% \mathrm{Ur}$ \\
\hline Condica viscosa (Freyer, 1831) & MEW & 3 & IX-X & $19-24^{\circ} \mathrm{C}$ & $69-86 \% \mathrm{Ur}$ \\
\hline Heliothis peltigera (D. \& S., 1775) & CEM & 1 & 27.IX.2008 & $19^{\circ} \mathrm{C}$ & $69 \%$ Ur. \\
\hline Helicoverpa armigera (Hübner, 1808) & SCO & 5 & II, VI, IX & $13-19^{\circ} \mathrm{C}$ & $69-81 \%$ Ur. \\
\hline Platyperigea terrea $($ Freyer, 1840$)$ & MES & 3 & III & $13-14^{\circ} \mathrm{C}$ & $84-85 \%$ Ur. \\
\hline Hoplodrina ambigua (D. \& S., 1775) & CEM & 9 & VI, IX-X & $18-20^{\circ} \mathrm{C}$ & $69-86 \% \mathrm{Ur}$. \\
\hline Spodoptera exigua (Hübner, 1808) & $\mathrm{SCO}$ & 26 & VII-XII & $12-24^{\circ} \mathrm{C}$ & $50-86 \%$ Ur. \\
\hline Sesamia cretica (Lederer, 1857) & AIM & 1 & 18.VI.2008 & $22^{\circ} \mathrm{C}$ & $81 \%$ Ur. \\
\hline Rusina ferruginea (Esper, 1785) & EUR2 & 1 & 04.VI.2008 & $18^{\circ} \mathrm{C}$ & $80 \%$ Ur. \\
\hline Mormo maura (Linnaeus, 1758) & TEM & 2 & 04.VI.2008; 27.IX.2008 & $18-19^{\circ} \mathrm{C}$ & $69-80 \% \mathrm{Ur}$. \\
\hline Phlogophora meticulosa (Linnaeus, 1758) & $\mathrm{EUM}+\mathrm{mac}$ & 2 & 19.III.2008; 07.VII.2008 & $14-28^{\circ} \mathrm{C}$ & $47-85 \%$ Ur. \\
\hline Callopistria juventina (Stoll, 1782) & PAL & 1 & 06.VIII.2008 & $23^{\circ} \mathrm{C}$ & $75 \%$ Ur. \\
\hline Chloantha hyperici (D. \& S., 1775) & EUR2 & 1 & 18.VI.2008 & $22^{\circ} \mathrm{C}$ & $81 \%$ Ur. \\
\hline Methorasa latreillei (Duponchel, 1827) & $\mathrm{EUM}+\mathrm{mac}$ & 15 & I, III-IV, VI, IX-XI & $12-20^{\circ} \mathrm{C}$ & $69-98 \%$ Ur. \\
\hline Xanthia sulphurago (D. \& S., 1775) & EUR6 & 1 & 15.X.2008 & $20^{\circ} \mathrm{C}$ & $86 \%$ Ur. \\
\hline Aporophyla nigra (Haworth, 1809) & EUM & 4 & XII & $10-13^{\circ} \mathrm{C}$ & $67-84 \%$ Ur. \\
\hline Mniotype solieri (Boisduval, 1840) & EUS & 32 & III-IV, VI, IX-XII & $12-22^{\circ} \mathrm{C}$ & $67-86 \%$ Ur. \\
\hline Mesapamea secalis (Linnaeus, 1758) & CAE1 & 1 & 23.VII.2008 & $22^{\circ} \mathrm{C}$ & $50 \%$ Ur. \\
\hline Nonagria typhae (Thunberg, 1784) & CAE & 1 & 18.VI.2008 & $22^{\circ} \mathrm{C}$ & $81^{\circ} \mathrm{Ur}$. \\
\hline Discestra trifolii (Hufnagel, 1766) & OLA & 1 & 10.I.2008 & $12^{\circ} \mathrm{C}$ & 98\%Ur. \\
\hline Aetheria bicolorata (Hufnagel, 1766) & ASE & 1 & 15.V.2008 & $17^{\circ} \mathrm{C}$ & $83 \%$ Ur. \\
\hline Hadena compta (D.\& S., 1775) & ASE & 7 & VIII-IX & $19-23^{\circ} \mathrm{C}$ & $69-76 \% \mathrm{Ur}$ \\
\hline Sideridis albicolon (Hübner, 1813) & ASE & 2 & 11.XI.2008 & $15^{\circ} \mathrm{C}$ & $76 \%$ Ur. \\
\hline Mythimna ferrago (Fabricius, 1787) & CAE & 2 & 06.VIII.2008 & $23^{\circ} \mathrm{C}$ & $75 \%$ Ur. \\
\hline Mythimna vitellina (Hübner, 1808) & $\mathrm{TEM}+\mathrm{mac}$ & 5 & V-VI & $17-22^{\circ} \mathrm{C}$ & $81-83 \%$ Ur. \\
\hline Mythimna obsoleta (Hübner, 1803) & OLA & 1 & 11.XI.2008 & $15^{\circ} \mathrm{C}$ & $76 \%$ Ur. \\
\hline Mythimna joannisi Boursin \& Rungs, 1952 & AFM & 6 & III-V, VIII & $14-23^{\circ} \mathrm{C}$ & $75-85 \%$ Ur. \\
\hline Mythimna putrescens (Hübner, 1824) & TEM & 16 & VIII-XI & $12-24^{\circ} \mathrm{C}$ & $69-86 \%$ Ur. \\
\hline Mythimna languida (Walker, 1858) & AIM & 10 & IV-V, X-XII & $10-19^{\circ} \mathrm{C}$ & $67-84 \%$ Ur. \\
\hline Mythimna l-album (Linnaeus, 1767) & PAL+India & 83 & I-XII & $9-28^{\circ} \mathrm{C}$ & $47-98 \%$ Ur. \\
\hline Mythimna sicula (Treitschke, 1835) & EUM & 8 & VI, XII & $10-22^{\circ} \mathrm{C}$ & $67-84 \%$ Ur. \\
\hline Mythimna prominens (Walker, 1856) & AIM & 22 & IV-V, VII-VIII, IX-XI & $12-28^{\circ} \mathrm{C}$ & $47-86 \%$ Ur. \\
\hline Mythimna loreyi (Duponchel, 1827) & AFM & 14 & IV-VI, IX, XII & $13-19^{\circ} \mathrm{C}$ & $67-83 \%$ Ur. \\
\hline Mythimna unipuncta (Haworth, 1809) & OLA & 1 & 06.XII.2007 & $13^{\circ} \mathrm{C}$ & $67 \%$ Ur. \\
\hline Orthosia incerta (Hufnagel, 1766) & ASE & 2 & 27.IX.2008 & $19^{\circ} \mathrm{C}$ & $69 \%$ Ur. \\
\hline Orthosia gothica (Linnaeus, 1758) & $\mathrm{CAE}$ & 1 & 04.III.2008 & $13^{\circ} \mathrm{C}$ & $84 \%$ Ur. \\
\hline Orthosia cerasi (Fabricius, 1775 ) & $\mathrm{CAE}$ & 1 & 04.III.2008 & $13^{\circ} \mathrm{C}$ & $84 \%$ Ur. \\
\hline Egira conspicillaris (Linnaeus, 1758) & CAE1 & 1 & 04.III.2008 & $13^{\circ} \mathrm{C}$ & $84 \%$ Ur. \\
\hline Axylia putris (Linnaeus, 1761) & ASE1 & 5 & IV, IX & $13-19^{\circ} \mathrm{C}$ & $69-79 \%$ Ur. \\
\hline Ochropleura leucogaster (Freyer, 1831) & MED5 & 2 & 06.II.2008; 18.VI.2008 & $13-22^{\circ} \mathrm{C}$ & $81 \%$ Ur. \\
\hline Noctua pronuba Linnaeus, 1758 & PAL & 10 & IV, VI, IX-X & $15-24^{\circ} \mathrm{C}$ & $79-82 \% \mathrm{Ur}$. \\
\hline Noctua interposita (Hübner, 1790) & TUE & 3 & IV-VI & $15-18^{\circ} \mathrm{C}$ & 79-83\%Ur. \\
\hline Noctua comes Hübner, 1813 & $\mathrm{EUM}+\mathrm{mac}$ & 6 & VI, IX, XI & $12-22^{\circ} \mathrm{C}$ & $69-81 \%$ Ur. \\
\hline Noctua janthina (D. \& S., 1775) & EUR & 1 & 07.VII.2008 & $28^{\circ} \mathrm{C}$ & $47 \%$ Ur. \\
\hline Noctua janthe (Borkhausen, 1792) & EUM & 1 & 06.VIII.2008 & $23^{\circ} \mathrm{C}$ & $75 \%$ Ur. \\
\hline Noctua interjecta Hübner, 1803 & EUR & 4 & $\mathrm{X}$ & $20^{\circ} \mathrm{C}$ & $86 \%$ Ur. \\
\hline Xestia c-nigrum (Linnaeus, 1758) & OLA & 3 & IX-X & $19-20^{\circ} \mathrm{C}$ & $69-86 \%$ Ur. \\
\hline Cerastis faceta (Treitschke, 1835) & MEW & 16 & I-XII & $9-13^{\circ} \mathrm{C}$ & $67-98 \%$ Ur. \\
\hline Peridroma saucia (Hübner, 1808) & $\mathrm{SCO}$ & 11 & I-II, IV, VI-VII, XI & $9-28^{\circ} \mathrm{C}$ & 47-98\%Ur. \\
\hline Agrotis puta (Hübner, 1803) & $\mathrm{CEM}+\mathrm{mac}$ & 71 & II-IV, IX-XI & $12-20^{\circ} \mathrm{C}$ & $69-86 \%$ Ur. \\
\hline Agrotis ipsilon (Hufnagel, 1766) & $\mathrm{COS}$ & 14 & I, IV-VI, IX-X, XII & $9-24^{\circ} \mathrm{C}$ & $67-84 \%$ Ur. \\
\hline Agrotis exclamationis (Linnaeus, 1758) & PAL & 3 & $\mathrm{X}$ & $20^{\circ} \mathrm{C}$ & $86 \%$ Ur. \\
\hline \multicolumn{6}{|l|}{ LYMANTRIIDAE } \\
\hline Lymantria dispar (Linnaeus, 1758) & ASE & 1 & 18.VI.2008 & $22^{\circ} \mathrm{C}$ & $81 \%$ Ur. \\
\hline
\end{tabular}




\begin{tabular}{|c|c|c|c|c|c|}
\hline Specie & Corotipo & $\begin{array}{c}\mathrm{N}^{\circ} \\
\text { esemplari }\end{array}$ & $\begin{array}{l}\text { Dati di } \\
\text { cattura }\end{array}$ & Temperatura & $\begin{array}{l}\text { Umidità } \\
\text { relativa }\end{array}$ \\
\hline Ocneria rubea (D. \& S., 1775) & EUS1 & 3 & VI, X & $18-20^{\circ} \mathrm{C}$ & $80-86 \%$ Ur. \\
\hline Laelia coenosa (Hübner, 1808) & CEM & 1 & 18.VI.2008 & $22^{\circ} \mathrm{C}$ & $81 \%$ Ur. \\
\hline \multicolumn{6}{|l|}{ NoLIDAE } \\
\hline$\overline{\text { Pseudoips prasinana (Linnaeus, } 1758)}$ & EUR2 & 2 & 27.IX.2008 & $19^{\circ} \mathrm{C}$ & $69 \%$ Ur. \\
\hline Earias insulana (Boisduval, 1833) & EUM & 4 & $\mathrm{~V}$ & $17^{\circ} \mathrm{C}$ & $83 \%$ Ur. \\
\hline \multicolumn{6}{|l|}{ ARCTIDAE } \\
\hline Eilema caniola (Hübner, 1808) & MES5 & 271 & IV-XI & $12-28^{\circ} \mathrm{C}$ & $47-86 \%$ Ur. \\
\hline Eilema pygmaeola (Doubleday, 1847) & EUW & 3 & VI, VIII-IX & $18-23^{\circ} \mathrm{C}$ & $69-80 \% \mathrm{Ur}$. \\
\hline Dysauxes famula (Freyer, 1836) & TUE & 184 & V-XI & $12-28^{\circ} \mathrm{C}$ & 47-86\%Ur. \\
\hline Phragmatobia fuliginosa (Linnaeus, 1758) & OLA & 28 & III-IV, VI-VII, IX-X & $13-28^{\circ} \mathrm{C}$ & $47-86 \% \mathrm{Ur}$. \\
\hline Cymbalophora pudica (Esper, 1784) & MED & 3 & IX-X & $19-20^{\circ} \mathrm{C}$ & $69-86 \%$ Ur. \\
\hline
\end{tabular}

Note

Dyspessa aculeata: specie localizzata e poco comune, la sua presenza è limitata ad alcune stazioni dell'Italia meridionale e della Sicilia (endemica). Vive in ambienti xerotermici e vola dal livello del mare fino a circa $800 \mathrm{~m}$ in una sola generazione da maggio a giugno (Bertaccini et al., 1997).

Nychiodes ragusaria: specie endemica di Basilicata, Calabria e Sicilia (dove è molto comune). Probabilmente bivoltina, vola da giugno a ottobre, dal litorale ai $1.550 \mathrm{~m}$ in località xerotermiche (Flamigni et al., 2007).

Calamodes subscudularia: vola dalla fine di settembre all'inizio di novembre. Molto abbondante nell'unica località calabrese nota (Fiumara del Trionto) (Scalercio \& Infusino, 2006), è invece rara e localizzata in Sicilia: i pochi dati esistenti riportano la presenza di esemplari isolati nell'area etnea (Turati, 1919) e una citazione dubbia per Monte Soro (Mariani, 1939). Le segnalazioni sono comunque tutte datate (la più recente risale a un esemplare della coll. Zangheri del 13.IX.1976) (Flamigni et al., 2007). Sempre secondo Flamigni et al. (2007), la specie in Sicilia sarebbe presente solo in montagna oltre i $600 \mathrm{~m}$. Questa segnalazione è la prima (per la Sicilia) in cui la specie si è presentata con un numero rilevante di esemplari. Visti anche i dati calabresi, si può ritenere che la specie prediliga le quote basse e gli habitat xerotermici, mentre la sua presenza a quote più elevate è da considerarsi occasionale.

Microloxia herbaria: specie xerotermofila, vola fra 0 e 400 m s.l.m., eccezionalmente più in alto. Abbastanza comune nelle aree costiere mediterranee. Larva polifaga (Hausmann, 2001).

Scopula minorata: specie fortemente xerotermofila, la sua distribuzione nel Mediterraneo è limitata alle zone costiere. Polivoltina e largamente polifaga (Hausmann, 2004).

Idaea filicata: specie molto comune nella macchia mediterranea, xerotermofila e polifaga. Predilige le quote basse, fino a $800 \mathrm{~m}$, ma è stata osservata fino ai $1.400 \mathrm{~m}$ s.l.m. (Hausmann, 2004).

Idaea seriata: specie estremamente comune e abbondante in Italia, soprattutto nel meridione. La larva è polifaga, gli adulti volano dal livello del mare ai 1.000 m s.l.m., eccezionalmente più in alto (Hausmann, 2004).

Rhodometra sacraria: specie ubiquitaria nel Mediterraneo, abbondante lungo le aree costiere. Larva polifaga su Polygonaceae, Anacardiaceae, Asteraceae, Chenopodiaceae, Rosaceae e altre (Hausmann, 2004).

Eupithecia dodoneata: in un primo momento identificati come E. lentiscata (Infusino, 2008), dopo essere stati sottoposti a barcoding analysis, si è potuto arrivare a una corretta identificazione degli esemplari.

Chloroclystis v-ata: ampiamente distribuita in tutta la regione paleartica, dal Portogallo al Giappone, verso nord si estende fino alla Norvegia, mentre il limite sud del suo areale sembra essere la costa algerina; in Italia si trova su tutto il territorio continentale. Karsholt \& Van Nieukerken (2011) la segnalano genericamente come presente in $\mathrm{Si}$ cilia, ma i dati di questa ricerca sono i primi certi per la regione (Infusino, 2008). La specie, al sud, è polivoltina e vola dall'inizio di aprile a metà ottobre. È stata rinvenuta in vari tipi di habitat, da 0 a oltre $2.000 \mathrm{~m}$ s.1.m. (Mironov, 2003).

Eublemma candidana: vola da maggio ad agosto, preferibilmente in località a ridosso del mare e delle spiagge. È specie xerotermofila (Berio, 1991).

Mythimna joannisi: la specie è un elemento faunistico tipico di paludi costiere e fragmiteti. È una 
specie poco comune ed estremamente localizzata. Questa è la seconda località nota in Sicilia (Parenzan, 1998).

Mythimna languida: specie subtropicale legata ad ambienti umidi e caldi, poco comune in Italia, dove si riscontra in pochissime località calabresi (Parenzan \& Scalercio, 1996) e siciliane (Bella et al., 1999; Russo et al., 2001; Parenzan \& Porcelli, 2006); questa è la terza località nota per la Sicilia.

Mythimna l-album: è specie comune e diffusa nelle località calde e umide, sale oltre i $1.600 \mathrm{~m}$ s.l.m. e i bruchi sono polifagi, soprattutto di erbe basse (Berio, 1985).

Agrotis puta: è una specie mesotermofila, frequente nelle zone costiere sabbiose o rocciose. I bruchi si nutrono di varie piante erbacee (Rakosy, 1996).

Laelia coenosa: la specie presenta due generazioni annue, in giugno e in agosto-settembre, con la seconda di norma più abbondante (Bertaccini et al., 1997). Si tratta di un rinvenimento di notevole interesse conservazionistico, trattandosi di una specie esigente dal punto di vista ambientale, soprattutto legata ad ambienti palustri litoranei. Tali ambienti sono sempre più rari a causa della forte antropizzazione delle coste. Conseguentemente, la distribuzione della specie è particolarmente frammentata in piccole popolazioni distanti tra loro. I Laghetti di Marinello rappresentano l'unica segnalazione per la Sicilia (Infusino et al., 2010).

Eilema caniola: specie presente e comune in tutta Italia. Polivoltina, frequenta ambienti diversi dal livello del mare ai $1.600 \mathrm{~m}$. Le larve si nutrono di licheni (Bertaccini et al., 1995).

Dysauxes famula: diffusa e comune nel centro-sud Italia, è una specie xerotermica che predilige gli ambienti calcarei. Si riscontra fino ai $1.000 \mathrm{~m}$ s.l.m. e le larve si nutrono di Tarassacum e altre piante basse (Bertaccini et al., 1997).

\section{Discussione}

La comunità di Macroeteroceri della Riserva Naturale Orientata "Laghetti di Marinello" è piuttosto povera di specie, ma questo risultato era prevedibile, date le condizioni abiotiche e biotiche estreme dell'area di studio. Infatti, alle temperature mediamente molto elevate si accompagna un substrato poco stabile, sottoposto ai continui rimaneggiamenti da parte del moto ondoso ordinario e delle mareggiate che possono modificare completamente la con- formazione del sistema dunale fino a far variare il numero e l'estensione dei laghetti; bisogna, poi, considerare la forte instabilità delle scogliere retrostanti con periodici franamenti che contribuiscono all'apporto di materiale, senza dimenticare la forte salinità che caratterizza l'habitat. A tutto questo si deve aggiungere che l'intero sistema dunale ha un'origine recentissima: non risultano esserci, infatti, notizie certe sulla sua esistenza prima del 1800 . Ciononostante, tali condizioni hanno comunque consentito l'instaurazione di una peculiare comunità floristica, seppur molto povera sia quantitativamente che qualitativamente. La scarsa quantità di specie e, soprattutto, la scarsa copertura vegetale fanno in modo che si rendano disponibili poche fonti alimentari sia per le larve che per gli adulti. Inoltre, essendo presenti per lo più piante a portamento erbaceo $\mathrm{o} a \mathrm{l}$ massimo arbustivo, scarseggiano anche ricoveri e ripari dal vento e dal forte irraggiamento solare.

Gli indici $\hat{\mathrm{S}}_{\text {Chaol }}$ e $\hat{\mathrm{S}}_{\mathrm{Chao} 2}$ hanno stimato rispettivamente a 186 e 191 il numero totale di specie ospitato dalla comunità. Entrambi gli indici concordano nell'attribuire una significativa efficacia ai campionamenti effettuati (rispettivamente 1' $86 \%$ e 1' $82 \%$ delle specie presenti sarebbe stato campionato), confermando di avere a disposizione un quadro abbastanza esaustivo della cenosi.

L'analisi della struttura di comunità evidenzia la presenza di una lunga coda di specie, di cui ben 47 rappresentate da un unico individuo e 24 rappresentate da 2 individui. Dal punto di vista quantitativo, la testa della comunità è caratterizzata, in ordine decrescente, da Eilema caniola, Eublemma viridula, Idaea filicata, Dysauxes famula e Idaea seriata, che da sole ne rappresentano il $42 \%$; di queste, solo $E$. caniola raggiunge il $10 \%$.

Eilema caniola ed Eublemma viridula sono certamente favorite dalla presenza delle loro piante nutrici, rispettivamente licheni ed Helichrysum italicum; in particolare, tra la scoglierea e il Lago Verde è presente un'associazione lichenica terricola che cresce all'interno di fitocenosi ascrivibili all'associazione Echinopo spinosissimi-Helichrysetum italici caratterizzata da varie specie appartenenti al genere Cladonia (P. Browne) (Licandro, com. pers.). Questi due Lepidotteri dimostrano un elevato adattamento alle condizioni climatiche, anche se con strategie diverse: E. caniola vola per tutto il periodo primaverile-estivo, con i picchi in corrispondenza dei valori 
medi di umidità e temperatura, facendo registrare una flessione in piena estate, quando le condizioni sono fortemente xerotermiche; E. viridula, al contrario, fa registrare i suoi picchi di densità proprio in corrispondenza dei valori di massima temperatura e minima umidità. Lo stesso vale anche per le altre specie citate, che, seppure polifaghe e piuttosto comuni, sono tutte strettamente xerotermofile.

Altre specie abbondanti sono state Mythimna lalbum, Agrotis puta, Scopula minorata, Rhodometra sacraria, Eublemma candidana e Gymnoscelis rufifasciata, che, insieme alle precedenti, rappresentano il $57 \%$ della comunità. Si tratta, soprattutto, di specie comuni e più o meno ubiquitarie (le prime due migratrici), ma anch'esse molto legate ad ambienti xerotermici.

Tutti gli indici di diversità calcolati concordano nel mostrare un basso livello di biodiversità ospitata (tab. 1). Ciò è dovuto, in massima parte, allo scarso numero di specie riscontrato, mentre l'indice di equiripartizione mostra un valore medio-alto, confrontabile con quello di ambienti con maggiore varietà biotica. Questi dati sono stati confrontati con quelli ottenuti in altri ambienti campionati con un metodo simile a quello scelto per questo lavoro (Scalercio et al., 2008a) (tab. 1). In particolare sono molto indicativi i valori relativi alla Fiumara Trionto, sia perché si tratta dell'ambiente che più si avvicina ai Laghetti di Marinello sia perché i dati si riferiscono a un solo anno di campionamento (mentre nelle altre aree si riferiscono a due anni) e sono, quindi, più direttamente confrontabili. In queste due aree gli indici di biodiversità sono simili, anche se quelli dei Laghetti di Marinello raggiungono valori più bassi. La comunità del Lago Angitola, posto a una quota molto bassa ma con un alto grado di stabilità e una vegetazione lussureggiante, mostra valori di biodiversità decisamente più elevati.

Un utile confronto è stato, inoltre, effettuato con i dati ottenuti da Bella et al. (1999) su un'aria umida della Sicilia sud-orientale, il Pantano Longarini, col metodo della caccia al lume per un periodo di due anni ma con cadenza mensile dei campionamenti. Il numero di specie (considerando solo quelle appartenenti alle superfamiglie oggetto di questa ricerca) catturate al Pantano Longarini è di 118, significativamente inferiore a quello relativo ai Laghetti di Marinello. Per quanto riguarda la similarità, sono stati calcolati gli indici di Sørensen e di Jaccard che fanno registrare, rispettivamente, valori di 0,44 e 0,28.

La macroeterocerofauna dei Laghetti di Marinello, seppure povera di specie, è del tutto peculiare per la presenza di alcune specie faunisticamente importanti come Mythimna joannisi, strettamente legata alle zone palustri costiere e conosciuta solo per un'altra località in Sicilia, Chloroclystis v-ata, nuova per la fauna siciliana, e soprattutto Laelia coenosa che, pur avendo un vasto areale di distribuzione, è molto rara e localizzata in ambienti lacustri costieri.

Le specie oligofaghe o monofaghe $(53 \%)$ sono più frequenti delle specie polifaghe (47\%), dimostrando un buon grado di specializzazione nella dieta larvale della maggior parte delle specie campionate e una loro diretta relazione con l'ambiente dei laghetti.

Come già riportato per altri ambienti (Scalercio, 2004; Scalercio \& Infusino, 2003, 2006; Scalercio et al., 2008a, 2008b), anche per i Laghetti di Marinello si ha un netto decremento del numero di individui che, fra la prima e la quarta ora di campionamento, arriva quasi a dimezzarsi. Il numero complessivo di specie nei sottocampioni di un'ora è molto simile, mentre per quelle esclusive di una delle quattro ore si assiste a un decremento con il procedere della notte, anche se non molto marcato; le specie esclusive della terza e quarta ora, comunque, non sono mai rappresentate da più di 3 individui.

Fenologia. Il numero di individui catturati durante l'inverno è piuttosto basso anche se mai nullo. Da marzo si assiste a un incremento delle catture che si accentua dalla fine di aprile fino a raggiungere $i$

Tab.1. Confronto tra i dati relativi ai principali indici di diversità riguardanti la comunità di Macroeteroceri dei Laghetti di Marinello e di altri quattro siti (da Scalercio et al., 2008a), dove S=numero di specie, H'obs=indice di Shannon, J'=Evennes su Shannon, $1 / \mathrm{D}=$ indice di Simpson inverso, $\alpha=$ Alpha di Fisher.

\begin{tabular}{l|c|c|c|c|c}
\hline & S & H'obs & J' & $\mathbf{1 / D}$ & $\alpha$ \\
\hline Laghetti Marinello & $\mathbf{1 6 0}$ & $\mathbf{3 , 7 9}$ & $\mathbf{0 , 7 5}$ & $\mathbf{2 2 , 5 9}$ & $\mathbf{3 9 , 2 0}$ \\
\hline Monte Cocuzzo & 298 & 4,51 & 0,79 & 51,36 & 67,43 \\
\hline Monte Curcio & 202 & 4,12 & 0,78 & 33,80 & 46,98 \\
\hline Lago Angitola & 294 & 4,29 & 0,75 & 35,98 & 57,71 \\
\hline Fiumara Trionto & 177 & 3,97 & 0,75 & 28,96 & 43,79 \\
\hline
\end{tabular}




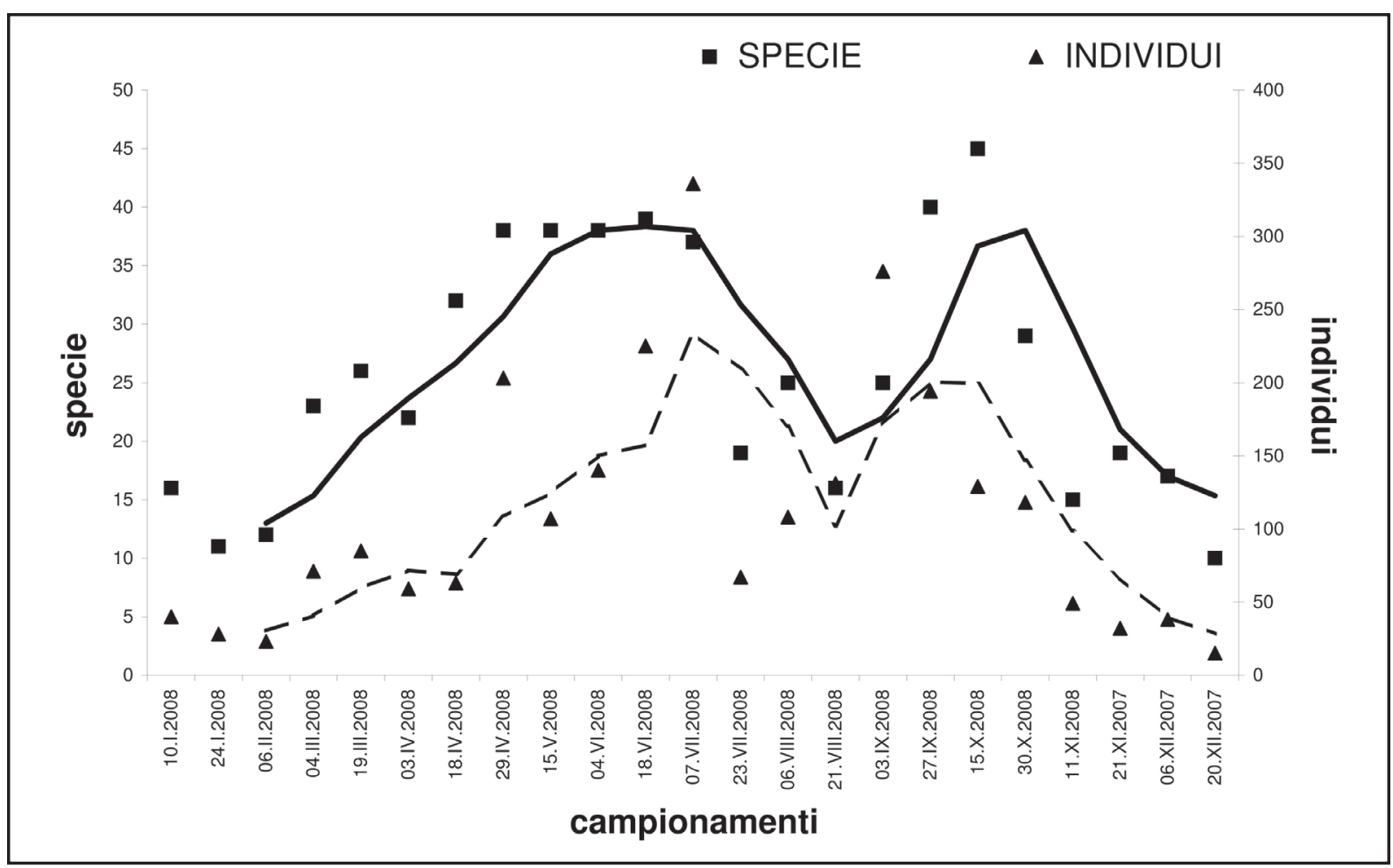

Fig. 2. Fenologia complessiva delle specie e degli individui catturati durante i campionamenti nel sito dei Laghetti di Marinello. Per migliorarne la leggibilità, nel grafico vengono riportate anche le linee di tendenza (media mobile su 3 rilievi) relative alle specie (linea continua) e agli individui (linea tratteggiata).

massimi all'inizio di luglio. Nell'ultima decade di luglio si assiste a un repentino calo, fino quasi ai livelli invernali, seguito da una ripresa in settembreottobre, quando le piogge mitigano l'aridità estiva e le temperature subiscono una lieve diminuzione. Con l'avanzare della stagione fredda, si ha un nuovo decremento fino ai già ricordati minimi invernali (fig.2). I picchi primaverile e autunnale sono dovuti in massima parte alla presenza di un nutrito contingente di specie bivoltine. Il numero di specie segue un andamento molto simile a quello degli individui, se si eccettua il picco autunnale che è molto più accentuato e addirittura supera, nei suoi valori massimi, quello primaverile. Quest'ultimo, però, ha una durata temporale decisamente maggiore di quello autunnale, mantenendosi a una quota superiore alle 32 specie dalla metà di aprile all'inizio di luglio.

Analisi Corologica. Le specie più frequenti sono state quelle ad ampia distribuzione oloartica, rappresentando il 50,31\% del totale (tab. 2). Le specie a diffusione mediterranea rappresentano il $31,05 \%$, mentre relativamente poche sono le specie europee che arri- vano al 14,29\%. L'analisi eseguita sugli individui mostra una frequenza dei corotipi oloartici che scende al $42,90 \%$, mentre quella dei corotipi europei e mediterranei sale rispettivamente al $21,49 \%$ e al $33,23 \%$. Fra le specie ad ampia diffusione e a distribuzione europea solo quelle distribuite in tutto il mediterraneo fanno registrare un incremento percentuale degli individui rispetto alle specie, mentre tutte le altre subiscono un deciso decremento. Questo mette ancora più in evidenza il carattere mediterraneo della comunità dei Laghetti di Marinello. Questa situazione è, chiaramente, il risultato delle condizioni climatiche estreme che si vengono a creare nel sistema dunale. La presenza dei laghetti, che pure contribuisce a mitigare l'aridità per gran parte dell'anno, non riesce comunque a compensare il forte irraggiamento e le temperature elevate della stagione estiva, oltre che la salinità dell'ambiente. Le specie a diffusione olopaleartica ed europea, che già si trovano al limite del loro areale, soffrono particolarmente queste condizioni, mentre le specie mediterranee riescono a sopportarle meglio o, addirittura, se ne avvantaggiano. 
Tab. 2. Analisi corologica. Si riportano il numero di specie e di individui appartenenti ai diversi corotipi. In parentesi sono riportate le percentuali.

\begin{tabular}{|c|c|c|}
\hline 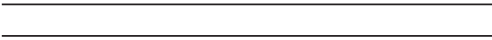 & Specie & Individui \\
\hline $\begin{array}{l}\text { Specie cosmopolite o subcosmopolite } \\
\text { COS+SCO }\end{array}$ & 7 (4) & $61(2)$ \\
\hline $\begin{array}{l}\text { Specie ad ampia diffusione oloartica } \\
\text { OLA+PAL+BAA } \\
\text { ASE + SIE } \\
\text { CAE } \\
\text { CEM+CAM } \\
\text { TUE+TEM } \\
\text { TUM } \\
\text { EUM } \\
\text { TOTALE }\end{array}$ & $\begin{array}{r}17(11) \\
12(7) \\
12(7) \\
16(10) \\
8(5) \\
9(6) \\
7(4) \\
81(50)\end{array}$ & $\begin{array}{r}161(6) \\
27(1) \\
26(1) \\
288(11) \\
287(11) \\
87(3) \\
212(8) \\
1088(43)\end{array}$ \\
\hline $\begin{array}{l}\text { Specie a distribuzione europea } \\
\text { EUR } \\
\text { EUS } \\
\text { EUW+ESW } \\
\text { TOTALE }\end{array}$ & $\begin{array}{r}9(6) \\
12(7) \\
2(1) \\
23(14)\end{array}$ & $\begin{array}{r}16(1) \\
301(12) \\
228(9) \\
545(21)\end{array}$ \\
\hline $\begin{array}{l}\text { Specie a distribuzione mediterranea } \\
\text { MED+MES } \\
\text { MEW+TIR } \\
\text { MEE } \\
\text { ADR } \\
\text { NAF+NAW } \\
\text { TOTALE }\end{array}$ & $\begin{array}{r}20(12) \\
10(6) \\
3(2) \\
1(1) \\
2(1) \\
36(22)\end{array}$ & $\begin{array}{r}436(17) \\
115(5) \\
60(2) \\
10(1) \\
19(1) \\
640(25)\end{array}$ \\
\hline $\begin{array}{l}\text { Specie afrotropicali e orientali } \\
\text { AIM } \\
\text { AFM } \\
\text { TOTALE }\end{array}$ & $\begin{array}{r}6(4) \\
5(3) \\
11(7)\end{array}$ & $\begin{array}{r}101(4) \\
97(4) \\
198(8)\end{array}$ \\
\hline $\begin{array}{l}\text { Elementi endemici italiani } \\
\text { APS }\end{array}$ & $3(2)$ & $5(1)$ \\
\hline TOTALE & $161(100)$ & $2537(100)$ \\
\hline
\end{tabular}

\section{CONCLUSIONI}

Le particolarità fenologiche e il netto carattere mediterraneo dell'intera cenosi caratterizzano molto bene la comunità di Macrolepidotteri Eteroceri dei Laghetti di Marinello, che sembra espressione diretta delle condizioni più naturali di questo ambiente ed è indice di un discreto stato di conservazione dell'habitat. Dal confronto con il Pantano Longarini, emerge ancora di più l'importanza di queste aree per la conservazione di alcune specie termofile o strettamente legate ad habitat umidi presenti in entrambi i siti (Mythimna joannisi, M. languida, Condica vi$s \cos a$ ); d'altro canto, i valori medio-bassi degli indici di similarità confermano la presenza, nei Laghetti di Marinello, di una comunità con caratteristiche proprie e distinte da altri ambienti similari. Il valore naturalistico di questa Riserva Naturale Orientata va al di là del semplice calcolo degli indici di diversità e deve essere letto nella particolarità del complesso dei suoi aspetti geomorfologici, climatici e biotici. Si tratta di un habitat effimero, molto instabile e dalle caratteristiche estreme, che hanno consentito l'instaurarsi di una flora e una fauna tipiche che contribuiscono notevolmente all'incremento della biodiversità complessiva del territorio sensu lato. Tanto più se si pensa che gli ambienti umidi e/o paludosi costieri, un tempo molto più numerosi, sono oggi estremamente vulnerabili perché ridotti a piccole o piccolissime "oasi", spesso assediate da stabilimenti balneari e abusivismo edilizio.

Il dato conservazionistico più rilevante è la presenza di Mythimna joannisi e Laelia coenosa, che da sola basterebbe a giustificare l'istituzione di un'area 
protetta in quanto trattasi di specie estremamente rare e localizzate.

\section{RINGRAZIAMENTI}

Doverosi ringraziamenti vanno al Dott. Alberto Zilli per il suo contributo nella determinazione del materiale, così come al Dott. Axel Hausmann che, inoltre, ci ha offerto la possibilità di effettuare la Barcoding Analysis sugli esemplari di Geometridae; al Dott. Gaspare Licandro per aver fornito i dati sulla flora della Riserva e per l'indispensabile supporto offerto durante i campionamenti; alla Dott.ssa Maria Letizia Molino per aver concesso le autorizzazioni a effettuare i prelievi all'interno della Riserva.

\section{BiBLIOGRAFIA}

Bella S., Russo P. \& Parenzan P., 1995 - Contributi alla conoscenza della lepidotterofauna della Sicilia. III. Bombici e Sfingi. Phytophaga, VI (1995): 85-109.

Bella S., Russo P. \& Parenzan P., 1999 - Contributi alla conoscenza della Lepidotterofauna siciliana. VII - I Lepidotteri Eteroceri del Pantano Longarini (Siracusa, Sicilia Sud-orientale). Phytophaga, IX: 15-37.

Berio E., 1985 - Fauna d'Italia. Lepidoptera Noctuidae. I. Generalità Hadeninae Cucullinae. Calderini, Bologna, 970 pp., 32 tavv.

Berio E., 1991 - Lepidoptera Noctuidae. II. Sezione quadrifide. Calderini, Bologna, 708 pp., 16 tavv.

Bertaccini E., Fiumi G. \& Provera P., 1995 - Bombici e Sfingi d'Italia (Lepidoptera Heterocera). Volume I. Natura-Giuliano Russo Editore, Bologna, 248 pp.

Bertaccini E., Fiumi G. \& Provera P., 1997 - Bombici e Sfingi d'Italia (Lepidoptera Heterocera). Volume 2. Natura-Giuliano Russo Editore, Bologna, 256 pp.

Cartabellotta D., Drago A., Lo Bianco B. \& Lombardo M., 1998 - Climatologia della Sicilia - Regione Siciliana, Assessorato Agricoltura e Foreste Gruppo IV - Unità di Agrometeorologia, Palermo.

Cernigliaro A., Di Benedetto R., Lombardo V., 1996 - Primo contributo alla conoscenza degli Eteroceri della Sicilia nordorientale: generi Leucania, Aletia, Sablia, Acantholeucania, Pseudaletia (Lepidoptera, Noctuidae). Bollettino della Società entomologica italiana, 128 (3): 158-188.

Flamigni C., Fiumi G. \& Parenzan P., 2007 - Lepidotteri eteroceri d'Italia Geometridae Ennominae I. Natura edizioni Scientifiche, Bologna, 384 pp., 16 tavv.

Giuliano F. \& Parenzan P., 1994 - Contributi alla conoscenza della lepidotterofauna siciliana II. I Macrolepidotteri dei Monti Nebrodi. Phytophaga, 5: 85-110.

Grillo N. \& Parenzan P., 1995 - Contributo alla conoscenza della Lepidotterofauna siciliana. I. Noctuidae. Phytophaga, V (1994): 51-83.

Grillo N. \& Parenzan P., 1996 - Contributi alla conoscenza della Lepidotterofauna siciliana. IV. Geometridae. Phytophaga, VI (1995): 111-129.

Hausmann A., 1993 - Contributo alla conoscenza della fauna siciliana: i rappresentanti della famiglia Geometridae presso la collezione dello Zoologische Staatssamlung di Monaco (Insecta: Lepidoptera). Il Naturalista Siciliano, S. IV, XVII (1-2): 83-101.

Hausmann A., 2001 - The Geometrid Moths of Europe. Volume I. Introduction, Archiearinae, Orthostixinae, Desmobathrinae, Alsophilinae, Geometrinae. Apollo Books, Stenstrup, 282 pp.

Hausmann A., 2004 - The Geometrid Moths of Europe. Volume II. Sterrhinae. Apollo Books, Stenstrup, 600 pp.

Heeger E., 1838 - Beitraege zur Schmetterlingskunde oder Abbildungen und Beschreibungen neuer Sicilianischer Schmetterlinge. Wien, Ed. J. P. Sollinger, 7 pp, 1 tav.

Infusino M., 2008 - Nuovi reperti di Geometridi (Lepidoptera) per la Sicilia. Il Naturalista Siciliano, S. IV, XXXII (3-4): 389-396.

Infusino M., Saitta S. \& Hausmann A., 2010 - Nuovi reperti di Lepidotteri Eteroceri per l'Italia e la Sicilia (Lepidoptera Geometridae, Lymantriidae). Bollettino della Società entomologica italiana, 142 (3): 129-134.

Infusino M., MacAione E. \& CALABRò C., 2008 - Relazioni tra le geodiversità e le biodiversità negli habitat lagunari della Riserva Naturale Orientata "Laghetti di Marinello" (Sicilia nord-orientale): particolarità sulla lepidotterofauna. I Convegno Nazionale "Geologia, cultura e sapori di Sicilia" - Messina 29-31 maggio 2008.

Karsholt O. \& Van Nieukerken E. J., 2011 - Lepidoptera, Moths. Fauna Europaea version 2.4, (eds. 2011). http://www. faunaeur.org

LeE S.M. \& ChaO A., 1994 - Estimating population size via sample coverage for closed capture-recapture models. Biometrics, 50: 88-90. 
Lederer J., 1852 - Versuch, die europäischen Lepidopteren. II. Abtheilung: Die Heteroceren. Verhandlungen des zoologischbotanischen Vereins in Wien, Abhandlungen, II: 65-126.

Licandro G., Marino P. \& Raimondo F. M., 2011 - Flora e vegetazione della Riserva Naturale Orientata "Laghetti di Marinello" (Sicilia nord-orientale). Informatore Botanico Italiano, 43 (2) 333-351.

Mariani M., 1939 - Fauna Lepidopterorum Siciliae. (Catalogo ragionato). Memorie della Società entomologica italiana, XVII (1938) (II): 129-187.

Mironov V., 2003 - Geometrid Moths of Europe. Vol. 4. Larentiinae II. Apollo Books, Stenstrup, 464 pp.

PARenZan P., 1994 - Proposta di una codificazione per una gestione informatica dei corotipi W-paleartici con particolare riferimento alla fauna italiana. Entomologica, 28: 93-98.

PARENZAN P., 1998 - Osservazioni preliminari sulla macrolepidotterofauna della riserva naturale di Vendicari (Siracusa) e prima segnalazione di Leucania palestinae Staudinger, 1897 (Lepidoptera, Noctuidae) per la fauna italiana. Il Naturalista Siciliano, S. IV, 22 (3-4): 355-364.

Parenzan P. \& Hausmann A., 1992 - Nuovi interessanti reperti di Geometridi (Lepidoptera) in Italia Meridionale, Entomofauna, 13 (8): 157-172.

Parenzan P. \& Porcelli F., 2006 - I macrolepidotteri italiani. Phytophaga, XV (2005-2006): 1-1051.

Parenzan P. \& Scalercio S., 1996 - Nuove segnalazioni di Nottuidi (Lepidoptera) per l'Italia meridionale. (Contributi alla conoscenza della Lepidotterofauna dell'Italia meridionale. XIX). Entomologica, 30: 105-133.

Parenzan P., Russo P. \& Bella S., 2002 - Eublemma pannonica (Freyer, 1840) (Lepidoptera, Noctuidae), specie nuova per la fauna di Sicilia e italiana. Contributi alla conoscenza della lepidotterofauna siciliana. IX. Entomologica, 36: 165-169.

Parenzan P., Bella S. \& Russo P., 2006 - Sette specie di Lepidotteri (Lycaenidae, Geometridae, Noctuidae) nuove per la fauna di Sicilia (Contributi alla conoscenza della lepidotterofauna siciliana. X). Entomologica, 39 (2005): 61-70.

RÁkosy L., 1996 - Die Noctuiden Rumäniens (Lepidoptera Noctuidae). Stapfia 46, 648 pp.

Ratnasingham S. \& Hebert P.D.N., 2007 - BOLD: The Barcode of Life Data System (www.barcodinglife.org). Molecular Ecology Notes, doi: 10.1111/j.1471-8286.2006.01678.x.

Russo. P., Bella S. \& Parenzan P., 2001 - Contributo alla conoscenza dei Nottuidi della Sicilia (Lepidoptera, Noctuidae). Phytophaga, XI: 11-85.

SCAlercio S. \& Infusino M., 2003 - I Macrolepidotteri di fosso Scuotrapiti, Lago dell'Angitola (Calabria, Italia Meridionale) (Lepidoptera). Phytophaga, XIII: 25-52.

SCALERCIO S., 2004 - Caratterizzazione della comunità dei macrolepidotteri eteroceri fototropici della pino-abetina di Monte Cocuzzo (Calabria, Italia). Atti del XIX Congresso Nazionale Italiano di Entomologia, Catania 10-15 giugno 2002, pp. 237-242.

SCAlercio S. \& Infusino M., 2006 - I Macrolepidotteri Notturni del Basso corso della fiumara del Trionto (Calabria, Italia Meridionale, Lepidoptera). Quaderni della Stazione ecologica del Museo civico di Storia naturale di Ferrara, 16: 181-204.

Scalercio S., Infusino M. \& Woiwood I. P., 2008a - Optimising the sampling window for moth indicator communities. Journal of Insect Conservation, doi:10.1007/s10841-008-9206-x.

Scalercio S., Infusino M. \& Tuscano J., 2008b - I Macrolepidotteri notturni della faggeta di Monte Curcio, Sila Grande (Calabria, Italia Meridionale) (Lepidoptera). Quaderni della Stazione ecologica del Museo civico di Storia naturale di Ferrara, 18: 5-19.

Schwingenschuss L., 1942 - Eine Falterausbeute aus Sizilien. I. Teil. Macrolepidoptera. Zeitschrift des Wiener Entomologen. Vereines, 27 (8): 177-184; (9): 222-224; (10): 244-249.

Turati E., 1919 - Nuove forme di Lepidotteri. Correzioni e note critiche. IV. Il Naturalista Siciliano, XXIII (7-12): 203-368, tavv. I-IV.

Zeller P. C., 1847 - Bemerkungen über die auf einer Reise, nach Italien, und Sicilien beobachteten Schmetterlinge. Isis, 40: (2): 121-159, (3): 213-233, (4): 284-308, (6): 401-457, (7): 481-521, (8): 561-594, (9): 641-673, (10): 721-771, (11): 801-859, (12): 881- 914.

Indirizzo degli autori:

M. Infusino, Dip. di Biologia Animale ed Ecologia Marina Università degli Studi di Messina, via F. Stagno d'Alcontres 31, I-98158 Messina ME, Italia.minfusino@unime.it

C. Calabrò, Dip. di Biologia Animale ed Ecologia Marina Università degli Studi di Messina, via F. Stagno d'Alcontres 31, I-98158 Messina ME, Italia.

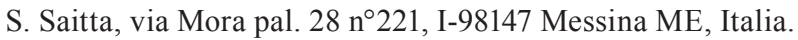

S. Scalercio, traversa San Proclo, 8, 87100 Cosenza, Italia. sscalercio@hotmail.com 